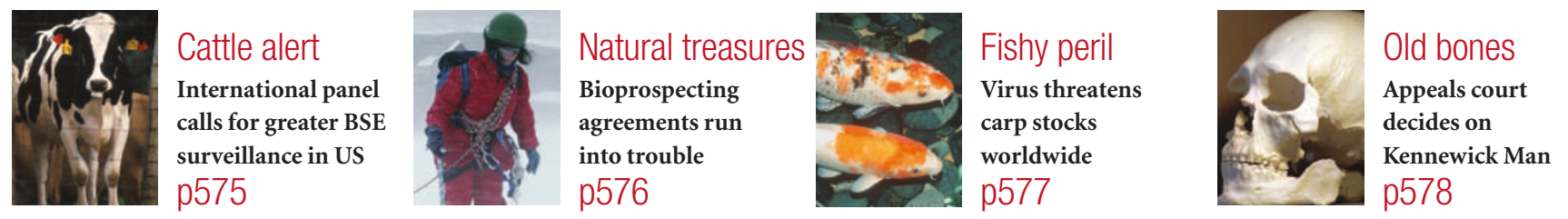

\title{
Virologists call for vaccination in bid to beat bird flu epidemic
}

\section{Alison Abbott}

Bird vaccination is needed to bring southeast Asia's epidemic of chicken flu under control, experts have concluded.

On 5 February the United Nations Food and Agriculture Organization (FAO) advised governments in affected areas that mass culling of birds (see Nature 427, $472-473$; 2004) is failing to halt the disease and that vaccination of targeted poultry flocks is required as well.

The advice followed an emergency meeting on vaccination strategies held by the FAO and other international bodies in Rome on 3-4 February. FAO officials are now working out the cost of vaccination, which would involve trained personnel administering what could be hundreds of millions of shots.

They will present a package of control plans, which include different levels of vaccination, at a regional FAO meeting in Bangkok later this month. The cost will vary enormously between commercial farms and remote villages, says Joseph Domenech, head of the FAO's animal health service - and it is unclear who will pay.

Chicken flu was relatively rare until 1997. But in the past six years more than 100 million birds have been infected by the disease or culled in its wake - half of them in southeast Asia this year - as farming becomes more intensive and reliant on the frequent movement of stock.

Until now, agricultural authorities have favoured culling over vaccination, in part because vaccinated animals can still harbour and shed infectious virus. Until recently it had not been possible to differentiate between infected and uninfected vaccinated animals, so vaccinated animals were still subject to trade bans.

But vaccination could help bring the latest outbreak under control by reducing the viral load in the environment, says Juan Lubroth, an avian-influenza expert at the FAO.

"Vaccination reduces the level of excretion of virus in infected animals, and also raises the threshold of viral load required for infectivity," explains Ilaria Capua, director of virology at the Veterinary Public Health Institute in Padua, Italy, whose vaccination

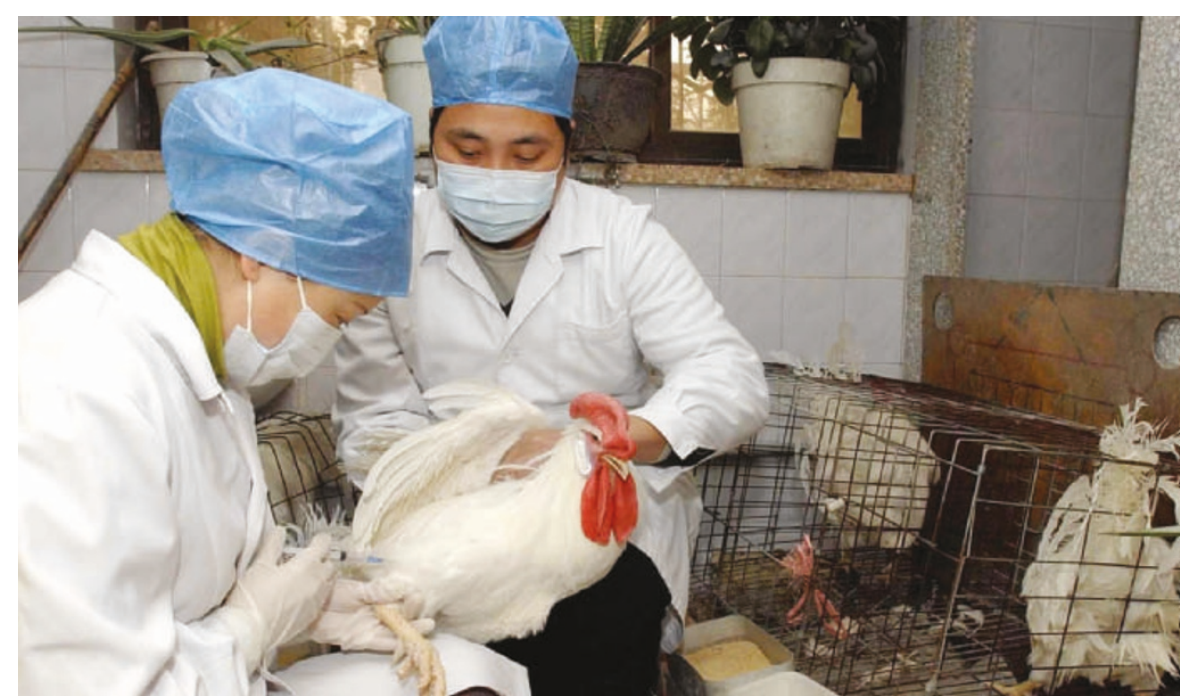

Chickens may be vaccinated in an effort to slow southeast Asia's avian flu epidemic.

strategy has already controlled two outbreaks of chicken flu in Italy.

Capua's strategy, known as DIVA (differentiating infected from vaccinated animals) allows chickens that are vaccinated, but still carrying the virus, to be identified by a molecular diagnostic test.

Avian influenza viruses have two key surface proteins. One is the haemagglutinin antigen, which has 15 subtypes, $\mathrm{H} 5$ and $\mathrm{H} 7$ being most frequently associated with pathogenicity. The other is neuraminidase $(\mathrm{N})$, which has nine subtypes and is less important in protection.

The vaccine used in a DIVA strategy is made from strains with the same $\mathrm{H}$ subtype as the circulating 'field' strain, but with a different $\mathrm{N}$ subtype. In the 2000-01 Italian outbreak of an H7N1 bird flu, a vaccine against an H7N3 strain was used. Vaccinated birds developed antibodies to the $\mathrm{H} 7$ and $\mathrm{N} 3$ proteins; birds infected with the circulating H7N1 strain also made antibodies to N1. A test to detect the anti-N1 antibodies was developed by scientists at Capua's institute, and the DIVA strategy was validated and approved by the Paris-based World Organisation for Animal Health and the European Union.

Confidence in the test, the operation of which was regularly checked by European
Commission officials, quickly led to the lifting of a trade ban on products from vaccinated, uninfected animals.

"We will consider using different vaccination strategies for different situations," says Lubroth. In some circumstances, the FAO will suggest using 'sentinel' birds unvaccinated birds kept within the vaccinated flocks - to monitor infection simply by the display of clinical symptoms. This is difficult to check, however, as farmers trying to hide infection could swap sick sentinel birds for healthy ones.

The bird vaccines cost just US2.5-5 cents per injected shot but even so a vaccination programme will be expensive as the number of vaccinations could run into billions. However, according to Capua, it could still be the best option if the flu continues to spread.

It would be hard to introduce the DIVA strategy in southeast Asia, however, as some affected countries have few facilities for testing for antibodies.

Capua is worried that some of the poorer countries in southeast Asia may not be able to monitor vaccinated birds carefully enough. "We have good vaccination tools to help the eradication effort, but if we don't use them properly it could make the situation worse," she warns. 